\title{
Biosynthesis of rhamnolipid by a Marinobacter species expands the paradigm of biosurfactant synthesis to a new genus of the marine microflora
}

\author{
Lakshmi Tripathi $^{1 *}$ (D, Matthew S. Twigg ${ }^{1}$, Aikaterini Zompra², Karina Salek ${ }^{3}$, Victor U. Irorere ${ }^{1}$, Tony Gutierrez ${ }^{3}$, \\ Georgios A. Spyroulias ${ }^{2}$, Roger Marchant ${ }^{1}$ and Ibrahim M. Banat ${ }^{1}$
}

\begin{abstract}
Background: In comparison to synthetically derived surfactants, biosurfactants produced from microbial culture are generally regarded by industry as being more sustainable and possess lower toxicity. One major class of biosurfactants are rhamnolipids primarily produced by Pseudomonas aeruginosa. Due to its pathogenicity rhamnolipid synthesis by this species is viewed as being commercially nonviable, as such there is a significant focus to identify alternative producers of rhamnolipids.

Results: To achieve this, we phenotypically screened marine bacteria for biosurfactant production resulting in the identification of rhamnolipid biosynthesis in a species belonging to the Marinobacter genus. Preliminary screening showed the strain to reduce surface tension of cell-free supernatant to $31.0 \mathrm{mN} \mathrm{m}^{-1}$. A full-factorial design was carried out to assess the effects of $\mathrm{pH}$ and sea salt concentration for optimising biosurfactant production. When cultured in optimised media Marinobacter sp. MCTG107b produced $740 \pm 28.3 \mathrm{mg} \mathrm{L}^{-1}$ of biosurfactant after $96 \mathrm{~h}$ of growth. Characterisation of this biosurfactant using both HPLC-MS and tandem MS showed it to be a mixture of different rhamnolipids, with di-rhamnolipid, Rha-Rha- $C_{10}-C_{10}$ being the most predominant congener. The strain exhibited no pathogenicity when tested using the Galleria mellonella infection model.

Conclusions: This study expands the paradigm of rhamnolipid biosynthesis to a new genus of bacterium from the marine environment. Rhamnolipids produced from Marinobacter have prospects for industrial application due to their potential to be synthesised from cheap, renewable feed stocks and significantly reduced pathogenicity compared to P. aeruginosa strains.
\end{abstract}

Keywords: Biosurfactant, Glycolipid, HPLC-MS, Marine bacteria, Marinobacter, Rhamnolipid

\section{Background}

Surfactant compounds possess both hydrophobic and hydrophilic moieties: they can modulate surface and interfacial tensions and are therefore widely utilised in a variety of different industries. Though many of these surfactant compounds are derived synthetically from

\footnotetext{
*Correspondence: I.tripathi@ulster.ac.uk

${ }^{1}$ School of Biomedical Sciences, Ulster University, Coleraine BT521SA, Northern Ireland, UK

Full list of author information is available at the end of the article
}

petrochemical sources, numerous microorganisms have been shown to synthesise surfactant compounds. Surfactant compounds produced from a biological source are termed biosurfactants and are generally viewed as being more sustainable and less toxic than their synthetically derived alternatives [1]. Marine microorganisms have been shown to be able to produce biosurfactants under extreme environments, caused by changes in salinity, increased UV exposure, limited nutrients, fluctuations in temperatures and $\mathrm{pH}$ [2-4]. Many marine bacterial species, commonly from oil-contaminated 
waters, have been reported to produce biosurfactants, and include members belonging to the genera Alcanivorax, Alteromonas, Pseudoalteromonas and Halomonas [5-7]. The biosurfactants they produce have the ability to solubilise hydrocarbons from the surrounding environment, which enhances the growth of indigenous bacteria capable of degrading aliphatic and polycyclic aromatic hydrocarbons (PAHs) [8]. These species therefore have tremendous industrial potential especially for application in microbial enhanced oil recovery (MEOR) and bioremediation purposes $[7,9,10]$. Furthermore, biosurfactant produced by psychrophilic marine bacteria are potentially exploitable in industrial processes for the preparation of biological detergents that are active at lower temperatures [11].

Particularly in the oligotrophic conditions of open ocean environments, marine bacteria have evolved to compete for the limited resources available to them. With respect to biosurfactants, they may be produced as secondary metabolites as, for example, to access hydrophobic growth substrates or to directly attack rival bacterial species competing for limited growth and energy sources [12]. In the latter case, biosurfactant compounds could be considered applicable for combatting pathogenic antibiotic-resistant microorganisms [13, 14]. Biosurfactants have also been shown to play an important role in biofilm development, the maintenance of biofilm structure and in substrate adhesion [15]. Abrogating the bacterium's ability to produce a biosurfactant could therefore disrupt biofilm growth with a multitude of potential applications including reducing infection risk to patients receiving implantable medical devices such as catheters [16, 17].

Biosurfactant compounds possess a wide array of molecular structures and are often classified based on their structure. One of the best studied groups of biosurfactants are the glycolipids, specifically rhamnolipids [10]. Rhamnolipids are composed of one or two rhamnose units linked in a 1,2-glycosidic linkage to two $\beta$-hydroxy fatty acids $(\beta$-OH-FA or $3-\mathrm{OH}-\mathrm{FA})$ ranging between 8 and 18 carbons in length. The most studied rhamnolipids are those produced by the Gram negative, opportunistic pathogen Pseudomonas aeruginosa [18]. Rhamnolipids have a broad range of potential applications in various industries, including for MEOR in the petroleum industry, as emulsifiers in the food and cosmetic industries, and as anti-microbial/therapeutically-active agents in the pharmaceutical industry [19]. Despite their versatile potential industrial applications, the exploitation of rhamnolipids has been limited due to the pathogenic nature of $P$. aeruginosa. To overcome this there has been an increased interest in the discovery of non-pathogenic rhamnolipid producers. Recent reports have shown rhamnolipid production by non-pathogenic species of Pseudomonas. For example, a non-pathogenic rhamnolipid-producing marine Pseudomonas sp. MCTG214(3b1) was shown to produce both mono and di-rhamnolipids [20]. An arctic marine bacterium identified as Pseudomonas fluorescence species was reported to synthesise five mono-rhamnolipid congeners [21]. Outside of the Pseudomonas genus, rhamnolipids have been shown to be synthesised by a number of non-pathogenic species of the genus Burkholderia [22, 23]. Discovery and isolation of novel non-pathogenic rhamnolipid producers is an attractive route to compete with the synthetic surfactants and meet future global biosurfactant requirements. Global estimates of microbial cell abundances in seawater range from $10^{4}$ to $10^{7}$ cells $/ \mathrm{mL}$, with an estimated average taxonomic diversity of 1000 species $/ \mathrm{mL}$ [24]. Collectively, this offers a significant opportunity to discover novel biosurfactant producers, including that produce rhamnolipids, and that are non-pathogenic [25]. Importantly for commercial exploitation, it is essential that the economics underlying the production of the biosurfactants is viable and able to compete with chemicallyderived surfactants in the global market. This needs to be achieved by reducing manufacturing costs and enhancing fermentation yields. There are numerous factors for optimising the process of biosurfactant production, including optimising the composition of the culture medium, $\mathrm{pH}$, dissolved oxygen levels during growth, culture agitation and incubation temperature. Statistical design of experiments (DoE) methods, such as full factorial design (FFD), have been shown to be an efficient and useful method to optimise biosurfactant production using a reduced number of experiments [26-28].

In this study, we investigated biosurfactant production in five bacterial strains isolated from coastal and offshore sites in the USA, Scotland and Norway, and all phylogenetically identified to belong to the genus Marinobacter. One of these strains, Marinobacter sp. MCTG107b, possessed phenotypic traits indicative of biosurfactant synthesis. The bioprocess factors for biosurfactant production in this strain were optimised for maximum production yield in shake-flask culture using FFD. Using high performance liquid chromatography-mass spectrometry (HPLC-MS) and tandem-MS, the chemical structure of the biosurfactants produced by this strain were analysed and confirmed to be rhamnolipids. This study reports the first description of rhamnolipid production by a Marinobacter and, importantly also, extends the paradigm of rhamnolipid production to a new bacterial genus which is recognised as ubiquitous in the marine environment and commonly associated with oil spills. 


\section{Materials and methods}

\section{Strains and culture conditions}

The marine bacteria used in this study were isolated from surface seawater samples collected from offshore location in the USA, UK and Norway (Table 1). The method of isolation has been previously described by Twigg et al. [20]. Following isolation, these strains were routinely cultured at $30{ }^{\circ} \mathrm{C}$ in $\mathrm{ZM} / 1$ medium which consists of $30 \mathrm{~g} \mathrm{~L}^{-1}$ sea salts (Sigma-Aldrich), $5 \mathrm{~g} \mathrm{~L}^{-1}$ Bacto Peptone (BD Biosciences), $1 \mathrm{~g} \mathrm{~L}^{-1}$ yeast extract (SigmaAldrich) and supplemented with trace elements and vitamins after autoclaving [29]. P. aeruginosa strain PAO1 was purchased from the ATCC (ATCC 15692) and was cultured at $37{ }^{\circ} \mathrm{C}$ in Nutrient Broth (Oxoid). Solid media plates used in this study were composed of appropriate culture media supplemented with $1.5 \%$ (w/v) agar (Sigma-Aldrich).

\section{Phylogenetic identification}

Genomic DNA (gDNA) was extracted from approx. $1 \times 10^{8}$ bacterial cells via a DNeasy Blood and Tissue Kit (Qiagen) used as per the manufactures instructions for Gram-negative bacteria. Extracted gDNA was quantified and assessed for purity by measuring absorbance at $260 \mathrm{~nm}$ and $280 \mathrm{~nm}$ using a Nanodrop 2000 spectrophotometer (Thermo Fischer). The 16S rRNA gene was then amplified using the Polymerase Chain Reaction (PCR) with the universal primers $9 \mathrm{bfm}$ and $1512 \mathrm{uR}$. PCR reactions contained $50 \mathrm{ng}$ of gDNA, $1.5 \mathrm{mM} \mathrm{MgCl}_{2}, 1 \times \mathrm{PCR}$ buffer (Thermo Fischer), $0.2 \mathrm{mM}$ dNTP mix (Thermo Fischer), $0.5 \mathrm{mM}$ of each primer (Thermo Fischer), and $2 \mathrm{U}$ of Taq DNA polymerase (Thermo Fischer). The PCR reaction was as follows: one cycle initial denaturation at $94{ }^{\circ} \mathrm{C}$ for $3 \mathrm{~min}$; 30 cycles of denaturation at $94{ }^{\circ} \mathrm{C}$ for $45 \mathrm{~s}$; annealing step at $52{ }^{\circ} \mathrm{C}$ for $30 \mathrm{~s}$; extension step at $72{ }^{\circ} \mathrm{C}$ for $90 \mathrm{~s}$; and one cycle final extension at $72{ }^{\circ} \mathrm{C}$ for $5 \mathrm{~min}$.
Following amplification, PCR products were separated on a $1 \%(\mathrm{w} / \mathrm{v})$ agarose gel made with TBE buffer (Thermo Fischer), and amplicons of approx. $1.5 \mathrm{~kb}$ were subsequently purified from the gel using a Wizard SV Gel and PCR Clean Up System (Promega). Amplified 16S rDNA was quantified and assessed for purity as above. The purified $16 \mathrm{~S}$ rDNA was sequenced using the Sanger method by Eurofins Genomics (Cologne, Germany) with primers $9 \mathrm{bfm}, 536 \mathrm{~F}, 907 \mathrm{R}$ and $1512 \mathrm{uR}[30,31]$. The resultant DNA sequences were compared to the NCBI nucleotide database using BLASTn.

\section{Phenotypic screening for biosurfactant production}

The five marine bacterial strains were screened for their ability to reduce surface tension and to emulsify oil in water. Bacterial cultures were centrifuged at $13,000 \times g$ for $15 \mathrm{~min}$ and the supernatant fractions (in triplicate) used to perform surface tension measurements at room temperature $\left(21^{\circ} \mathrm{C}\right)$ according to the Du Noüy ring method using a K10ST A KRÜSS KIOT Tensiometer (Krüss) [32]. The surface tension of sterile ZM/1 media supplemented with $1 \%(\mathrm{v} / \mathrm{v})$ rapeseed oil (Sigma-Aldrich) was also measured as a comparative control. To evaluate for emulsification, the emulsification index (EI) of the supernatant fractions was measured by adding $2 \mathrm{~mL}$ of the supernatant to an equal volume of kerosene and vortexing at high speed for $2 \mathrm{~min}$. The stability of resultant emulsions was observed after $24 \mathrm{~h}$ settlement. The $\mathrm{EI}_{24}$ (i.e. EI after $24 \mathrm{~h}$ ) was calculated as a percentage of the height of the emulsified layer to the total height of the liquid prior to emulsification by vortexing [33]. As a control for comparison, sterile ZM/1 medium was used.

\section{Optimization of growth conditions}

The growth of MCTG107b in different physical and media conditions was investigated. For this, shake flask

Table 1 Phylogenetic identification and biosurfactant phenotypic screening results for each bacterial strain

\begin{tabular}{|c|c|c|c|c|c|c|}
\hline \multirow[t]{2}{*}{ Strain } & \multirow[t]{2}{*}{ Origin } & \multirow{2}{*}{$\begin{array}{l}\text { BLASTn identification } \\
\text { (against NCBI database) }\end{array}$} & \multirow{2}{*}{$\begin{array}{l}\text { GeneBank } \\
\text { accession } \\
\text { number }\end{array}$} & \multirow{2}{*}{$\begin{array}{l}\text { Sequence } \\
\text { similarity } \\
(\%)\end{array}$} & \multicolumn{2}{|c|}{ Phenotypic screening } \\
\hline & & & & & $\mathrm{ST}\left(\mathrm{mN} \mathrm{m}^{-1}\right)^{\mathrm{a}}$ & $\mathrm{El}_{24 \mathrm{~h}}(\%)^{\mathrm{b}}$ \\
\hline MCTG106 & $\begin{array}{l}\text { Coastal surface water, Oregon, Washing- } \\
\text { ton State, USA }\end{array}$ & Marinobacter sp. NP1383C-30R & MK894600 & 100 & $54.63 \pm 3.5$ & $40 \pm 1.5$ \\
\hline MCTG4b & $\begin{array}{l}\text { Laboratory culture of Thalassiosira weiss- } \\
\text { flogii strain CCMP } 1052 \text { isolated from } \\
\text { Oslo Fjord, Norway }\end{array}$ & Marinobacter sp. Set72 & MK894835 & 99 & $38.5 \pm 0.6$ & $42 \pm 2.0$ \\
\hline MCTG167 & Phytoplankton net tow, Oban, UK & Marinobacter sp.T23 & MK894854 & 100 & $61.55 \pm 0.1$ & $\mathrm{~N} / \mathrm{A}$ \\
\hline MCTG161(2C3) & Phytoplankton net tow, Oban, UK & Marinobacter adhaerens HP15 & MK894872 & 99 & $60.0 \pm 0.5$ & $45 \pm 2.0$ \\
\hline MCTG107b & $\begin{array}{l}\text { Coastal surface water, Oregon, Washing- } \\
\text { ton State, USA }\end{array}$ & Marinobacter sp. R-28768 & MK578516 & 100 & $31.0 \pm 0.5$ & $40 \pm 1.8$ \\
\hline
\end{tabular}

Bacteria were identified by $16 \mathrm{~S}$ rDNA gene sequencing. Surface tension values $\left(\mathrm{mN} \mathrm{m}^{-1}\right)$ and $\mathrm{El}_{24}(\%)$ were obtained from cell-free supernatant samples of cultures incubated for $96 \mathrm{~h}$

a $\mathrm{ST}$ and ${ }^{\mathrm{b}} \mathrm{El} 24 \mathrm{~h}$ of sterile $\mathrm{ZM} / 1$ medium was $58 \mathrm{mNm}^{-1}$ and $0 \%$, respectively 
experiments were carried out in $1 \mathrm{~L}$ Erlenmeyer flasks containing $90 \mathrm{~mL}$ of $\mathrm{ZM} / 1$ medium and inoculated $(10 \%$ $\mathrm{v} / \mathrm{v}$ ) with a seed culture grown under the standard conditions described earlier. For the carbon source, glucose $1 \%$ $(\mathrm{w} / \mathrm{v})$ final concentration was used in all experiments. All flasks were incubated in a rotary orbital incubator set at $200 \mathrm{rpm}$. However, physical and media conditions tested included various concentrations of sea salts $(5,10,20,30$ and $\left.40 \mathrm{~g} \mathrm{~L}^{-1}\right)$, temperature $\left(25,28,30\right.$ and $\left.37^{\circ} \mathrm{C}\right)$ and $\mathrm{pH}$ $(4.0,5.5,7,8.5)$. Samples of the culture medium in these various experiments were taken at various time points for optical density (OD) measurements at $600 \mathrm{~nm}$ to monitor the growth of the cells.

\section{Optimisation of growth media for biosurfactant production}

The effect of sea salt concentration and $\mathrm{pH}$ for enhancement of biosurfactant production by MCTG107b was carried out by FFD, using surface tension, (measured as described previously), and biosurfactant yield, (measured gravimetrically), as response variables. These experiments were carried in shake flask culture using $\mathrm{ZM} / 1$ medium supplemented with $1 \%(\mathrm{v} / \mathrm{v})$ rapeseed oil with various concentrations of sea salts $\left(5\right.$ to $40 \mathrm{~g} \mathrm{~L}^{-1}$ ) and $\mathrm{pH}$ (5.5 to 8.5) according to the experimental designs (Additional file 1: Table S1). In total, 14 experiments were performed $\left(2^{2}\right.$ FFD with 8 assays and 6 replicates at the centre point).

\section{Biosurfactant extraction and purification}

Biosurfactant compounds were extracted and purified from 3-L cultures of strain MCTG107b when grown in optimised ZM/1 medium supplemented with $1 \%$ (v/v) rapeseed oil using a $5.0 \mathrm{~L}$ Biostat B bioreactor (Sartorius Stedim) equipped with a mechanical foam separator. The reactor vessel was inoculated $(10 \% \mathrm{v} / \mathrm{v})$ with a MCTG107b seed culture grown to exponential phase in $\mathrm{ZM} / 1$ supplemented with $1 \%(\mathrm{w} / \mathrm{v})$ glucose at $30{ }^{\circ} \mathrm{C}$ and $200 \mathrm{rpm}$. Internal temperature of the culture was maintained at $30{ }^{\circ} \mathrm{C}$ throughout the growth cycle. Stirrer speed and aeration varied between 300 and $600 \mathrm{rpm}$ in order to maintain $\mathrm{DO}_{2}$ levels at $50 \%$. Cultures were incubated for $96 \mathrm{~h}$; during growth, dissolved oxygen and $\mathrm{pH}$ were continually monitored and samples were taken at $24 \mathrm{~h}$ intervals to monitor growth and BS.

At the termination of the culture $(96 \mathrm{~h})$, the biosurfactants were extracted using liquid phase extraction. For this, the culture volume was first centrifuged $(13,000 \times g$; $15 \mathrm{~min}$ ) and then the supernatant collected and acidified to $\mathrm{pH} 2.0$ with $1 \mathrm{M} \mathrm{HCl}$ (Sigma-Aldrich) prior to extraction three times with an equal volume of ethyl acetate (Sigma-Aldrich). The organic phase was separated and dried using $\mathrm{MgSO}_{4}$ (Sigma-Aldrich), filtered and rotary evaporated under vacuum at $40{ }^{\circ} \mathrm{C}$ to obtain a crude extract [34]. Crude extracts were then purified by Solid Phase Extraction (SPE) using Strata SI-1 Silica $(55 \mu \mathrm{m}$, $70 \AA$ A) Giga tubes (Phenomenex). Purified BS extracts were gravimetrically assessed and stored at $4{ }^{\circ} \mathrm{C}$ for further analysis [35].

\section{Chemical analysis of biosurfactant compounds}

Biosurfactant compounds extracted from strain MCTG107b were initially analysed using the orcinol method [34]. To each $100 \mu \mathrm{L}$ sample, $900 \mu \mathrm{L}$ of a solution containing $0.19 \%(\mathrm{w} / \mathrm{v})$ orcinol (Sigma-Aldrich) in $53 \% \mathrm{H}_{2} \mathrm{SO}_{4}$ (Sigma-Aldrich) was added. Samples were then heated to $80{ }^{\circ} \mathrm{C}$ for $30 \mathrm{~min}$, after which the samples were cooled to room temperature. The absorbance of the samples was measured at $421 \mathrm{~nm}$. The concentration of glycolipid present in the samples were calculated to those generated using a standard rhamnose at concentrations of $0-100 \mu \mathrm{g} \mathrm{mL}^{-1}$ [36].

Individual biosurfactant congeners were identified in the SPE purified extract by a UHPLC system with RS Diode Array detector (ThermoFisher Scientific) in conjunction with the amaZon SL dual funnel Ion Trap spectrometer LCMS system (Bruker). An analytical column of Acclaim RSLC, $120 \mathrm{C} 18,2.2 \mu \mathrm{m} 120 \AA(2.1 \times 100 \mathrm{~mm})$ (ThermoFisher Scientific) was used for analysis. The gradient elution sequence used was as follows: $20 \% \mathrm{~B}$ to $100 \%$ in $30 \mathrm{~min}, 100 \%$ B for $10 \mathrm{~min}, 20 \% \mathrm{~B}$ in $5 \mathrm{~min}$. Solvents $\mathrm{A}=\mathrm{H}_{2} \mathrm{O}(0.1 \%$ TFA), $\mathrm{B}=\mathrm{AcCN}$ (0.1\%TFA). The sample injection volume used was $10 \mu \mathrm{L}$. Spectra were acquired in the positive mode from $\mathrm{m} / \mathrm{z} 200$ to 2000 . Tandem-MS was performed using Thermos System LC P4000 (ThermoFisher Scientific) coupled to a LCQ classic MATT ion trap mass spectrometer (ThermoFisher Scientific) equipped with a $150 \times 4.6 \mathrm{~mm}$ Kinetex $5 \mu \mathrm{M}$ F5 100 A LC column. HPLC-grade water and analyticalgrade acetonitrile were used as mobile phase. The sample injection volume was $5 \mu \mathrm{L}$ and the spectra were acquired in the negative mode from $m / z 175$ to 700 . The fragmentation of the molecules was done with helium gas at the normalised $40 \%$ collision energy with the activation $q$ value of 0.25 .

\section{Galleria mellonella infection model}

Virulence assessment of strain MCTG107b was carried out using the G. mellonella infection model [38]. A comparative positive control for these experiments was P. aeruginosa PAO1 (ATCC 15692). Using the Miles and Misra method [37], viable count (CFU) and OD $600 \mathrm{~nm}$ were correlated throughout the growth cycles of both strains. Following this, $10 \mathrm{~mL}$ of stationary phase culture 
was centrifuged $(10,000 \times g ; 20 \mathrm{~min})$ and the pelleted cells washed in sterile phosphate buffered saline (PBS). The washed cells were re-suspended to OD $600 \mathrm{~nm} 0.4$ in PBS to a concentration of $5 \times 10^{4} \mathrm{CFU} \mathrm{mL} \mathrm{m}^{-1}$. G. mellonella larvae (Pets at Home, Belfast) of approx. $20 \mathrm{~mm}$ in length and $200 \mathrm{mg}$ in weight were selected and $20 \mu \mathrm{L}$ of either bacterial sample (1000 CFU) or PBS (negative control) was injected into the posterior pro-leg of individual larvae ( $\mathrm{n}=10$ per experimental group). Injection was carried out using a $0.30 \mathrm{~mm}(30 \mathrm{G}) \times 8 \mathrm{~mm}$ hypodermic needle (BD). Immediately following injection, the larvae were incubated at $37{ }^{\circ} \mathrm{C}$ and observed at set time points during the course of a $48 \mathrm{~h}$ period. At each time point, individual larva was recorded as either live or dead. The experiment was performed on three independent occasions which gives a total of $\mathrm{n}=30$ per experimental group $[20,38]$.

\section{Statistical analysis and data availability}

Statistical analysis of bacterial growth experiments was carried out in GraphPad Prism V.7 using a one-way ANOVA followed by Tukey's post hoc testing; the significance of the results was tested at $p<0.05$ level. The data obtained from FFD experiments were subjected to statistical analysis by TIBCO Statistica software version; the significance of the results was tested at $p<0.05$ level. All sequence data was submitted to GenBank (NCBI, USA) and the assigned accession numbers of strains are given in Table 1.

\section{Results}

\section{Strain identification and initial phenotypic screening}

Five marine bacterial isolates-MCTG107b, MCTG4b, MCTG106, MCTG167 and MCTG161(2c3) - were investigated for biosurfactant production. BLASTn analysis of partial 16S rRNA gene sequences from these strains showed $>99 \%$ similarity to the genus Marinobacter (Table 1). Following phenotypic screening, strains which reduced the surface tension of culture medium to below $35 \mathrm{mN} \mathrm{m}^{-1}$ and/or produced a stable emulsion after $24 \mathrm{~h}$ were considered as potential biosurfactant(s) producers (Table 1). Strain MCTG167 was unable to form a stable emulsion or to significantly reduce the surface tension when compared to un-inoculated medium controls $\left(58 \mathrm{mN} \mathrm{m}^{-1}\right)$. Strain MCTG106 and MCTG161(2c3) also showed no significant reduction in surface tension, however both strains were able to form stable emulsions after $24 \mathrm{~h}$. Strain MCTG4b emulsified kerosene with an $\mathrm{EI}_{24}$ of $40 \%$, whereas it reduced the ST of supernatant fractions to $38.5 \mathrm{mN} \mathrm{m}^{-1}$. Strain MCTG107b showed the highest surface activity, significantly reducing the surface tension of the culture broth to $31 \mathrm{mN} \mathrm{m}^{-1}$, and producing stable emulsions with kerosene $\left(\mathrm{EI}_{24}\right.$ of $\left.40 \%\right)$. Therefore, Marinobacter sp. MCTG107b was selected for further study.

\section{Bacterial growth of Marinobacter sp. MCTG107b}

To determine optimal conditions for the growth of Marinobacter sp. MCTG107b, and with a view to optimising its production of biosurfactant, growth was monitored in different physical and media conditions that included evaluating different salinities, $\mathrm{pH}$, temperatures and nitrogen sources. Marinobacter sp. MCTG107b grew optimally at a range of salinity concentrations, from 5.0 to $40 \mathrm{~g} \mathrm{~L}^{-1}$ sea salts. In medium containing no added sea salts, no growth was observed. Strain MCTG107b grew optimally within a $\mathrm{pH}$ range of 5.5 to 8.5 , whereas it was significantly inhibited under more acidic conditions $(\mathrm{pH}$ 4.0). The strain grew optimally at temperatures ranging from 25 to $37^{\circ} \mathrm{C}$ (Additional file 1: Fig. S1).

\section{Optimization of culture conditions for biosurfactant production}

To optimise the media composition for maximal biosurfactant production by strain MCTG107b, the effects of salinity and $\mathrm{pH}$, as well as the interaction between these variables was assessed by applying FFD, $2^{2}$. Compared to $\mathrm{pH}$, salinity was observed to be the most important factor affecting the reduction of surface tension when this was measured for cell-free culture supernatant fractions. The surface tension was found to vary between 30.5 to $40.2 \mathrm{mN} \mathrm{m}^{-1}$ (Additional file 1: Table S1). Experimental results were used to generate two equations that modelled the relation between $\mathrm{pH} /$ salinity and the outputs biosurfactant yield and surface tension. According to the response values obtained from the designed experiments, the following regression equations were obtained for both biosurfactant yield (1) and surface tension (2):

$$
\begin{aligned}
\text { Yield }= & 22.17262+39.88095 * p H(1)+36.60714 \\
& * \text { Salts }(2)-4.64286 * p H * \text { Salts }(1 \text { by } 2)
\end{aligned}
$$

$$
\begin{aligned}
\text { Surface Tension }= & 50.03036-1.70714 * p H(1) \\
& -0.55690 * \text { Salts }(2)+0.05476 \\
& * p H * \text { Salts }(1 \text { by } 2)
\end{aligned}
$$

Significance of the present model was validated through analysis of variance $(p \leq 0.05)$ (Additional file 1 : Table S2). The observed values for biosurfactant yield were significantly close to those determined by the model $\left(R^{2}=0.996\right)$. The observed and the predicted values for surface tension modelled here also demonstrated that the 
experimentally observed values were significant to those determined by the model $\left(R^{2}=0.958\right)$. Results from the FFD analysis for the outputs of biosurfactant yield and surface tension were expressed as 3D response surface plots showing the relationship between independent and dependent variables (Fig. 1). Increased salinity significantly and positively influenced biosurfactant yield. Our model demonstrated a significant relationship between biosurfactant yield and the two variables tested (salinity and $\mathrm{pH}$ ). We modelled an increased biosurfactant yield when $\mathrm{pH}$ was low and salinity was increased from the central to the highest level. The increase in salinity positively influenced biosurfactant yield, in a statistically significant way (Fig. 1a). Similarly, the model showed that an increase in $\mathrm{pH}$ did not cause major impacts on surface tension, whereas an increase in salinity from the central to highest point led to a decrease in surface tension. The results of reduction in superficial tension agree with the biosurfactant yield. However, the 3D response surface plots of both dependent responses are not a coincident. Since, the increase in salinity concentration negatively influenced, in a statistically significant way, the increase in biosurfactant production, leading to lower surface tension (Fig. 1b). These data demonstrate salinity and $\mathrm{pH}$ are critical factors that markedly affected the production yield of biosurfactant. Our model showed maximum production of biosurfactant at a $\mathrm{pH}$ 5.0-6.8 and salinity $22.5-40.0 \mathrm{~g} \mathrm{~L}^{-1}$, resulting in a predicted yield of between 460 and $800 \mathrm{mg} \mathrm{L}^{-1}$. The optimised medium with a sea salt concentration of $30 \mathrm{~g} \mathrm{~L}^{-1}$ and $\mathrm{pH} 6.5$ was therefore chosen for all subsequent experiments with strain MCTG107b.

\section{Growth and biosurfactant production by Marinobacter sp. MCTG107b in a bioreactor}

Based on our results above, an optimised culture medium was used to produce biosurfactant from Marinobacter sp. MCTG107b employing a bench-scale 5.0 L bioreactor. Biomass, culture $\mathrm{pH}$ and surface tension were monitored continually throughout the growth cycle. The cell concentration at the time of inoculation $(t=0 h)$ was $2.02 \times 10^{6} \mathrm{CFU} \mathrm{mL} \mathrm{m}^{-1}$. An exponential growth phase was maintained for the first $24 \mathrm{~h}$, followed by a stationary phase from 24 to $96 \mathrm{~h}$, and the cell concentration reaching $6.46 \times 10^{9} \mathrm{CFU} \mathrm{mL} \mathrm{m}^{-1}$ by the end of the fermentation (Fig. 2). The $\mathrm{pH}$ of the culture was observed to fall during the course of the exponential growth phase and then remained moderately constant during the stationary phase. A similar pattern was observed for the surface tension that was measured for cell-free supernatant samples

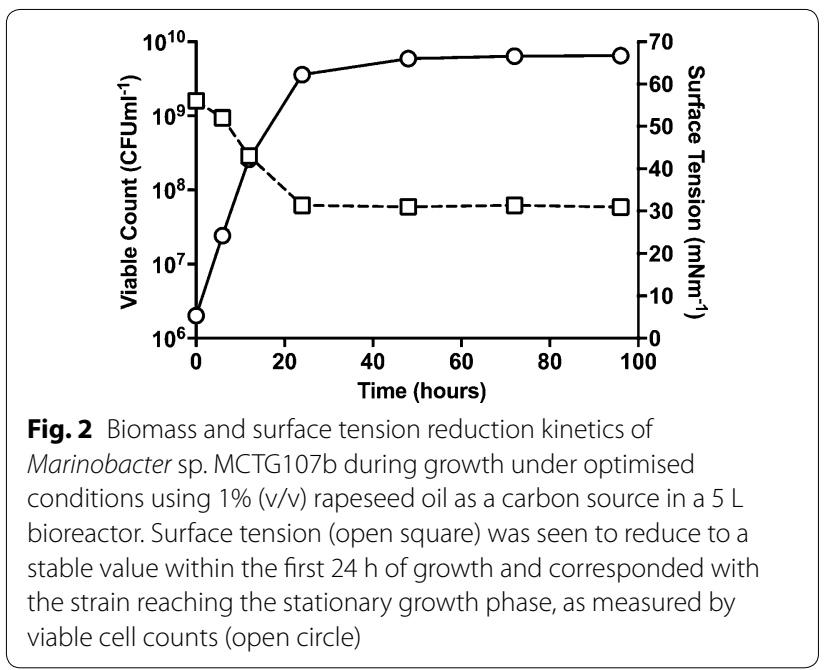

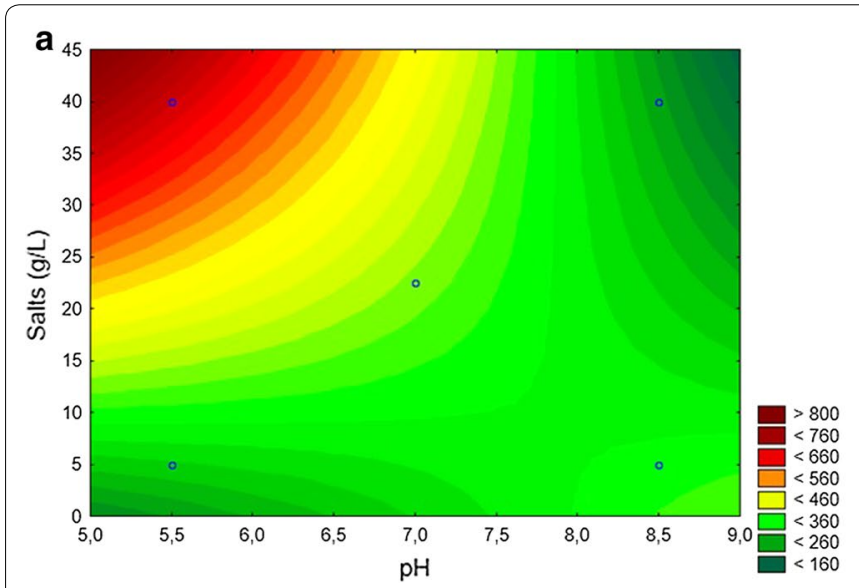

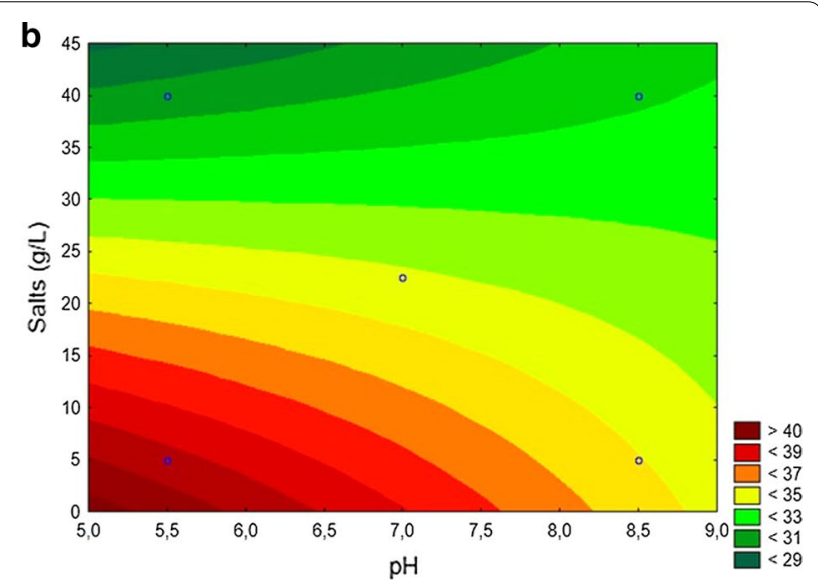

Fig. 1 Three-dimensional response surface plot modelling the effect of varying media $\mathrm{pH}$ and salt concentration on $\mathbf{a}$ biosurfactant yield and $\mathbf{b}$ cell-free supernatant surface tension. The different coloured areas of these plots represent various bands for either predicated yield or predicted surface tension. The values of each band are provided in the key next to each panel 
over the course of the growth phase. The strain achieved the lowest surface tension value $\left(31 \pm 0.7 \mathrm{mN} \mathrm{m}^{-1}\right)$ after $24 \mathrm{~h}$ of fermentation and then remained almost constant until the end of the fermentation (Fig. 2). When assessed gravimetrically, the mean biosurfactant yield obtained by liquid phase extraction and SPE purification of the cellfree supernatant volume from replicate $96 \mathrm{~h}$ bioreactor cultures of Marinobacter sp. MCTG107b was $740 \mathrm{mg} \mathrm{L}^{-1}$ $\left( \pm 28.3 \mathrm{mg} \mathrm{L}^{-1}\right)$.

\section{Chemical characterisation of the biosurfactant produced by Marinobacter sp. MCTG107b}

The traits measured for biosurfactant production (i.e. surface tension reduction and emulsification) were indicative that Marinobacter sp. MCTG107b produces a glycolipid biosurfactant. To confirm this, Orcinol assays followed by HPLC-MS analysis were carried out on samples obtained from the bioreactor cultures. Orcinol assays performed with cell-free supernatant samples indicated the presence of glycolipids at $150 \mu \mathrm{g} \mathrm{mL}$ of culture. The production and presence of glycolipids was further investigated by mass spectrometric analysis. The identification of glycolipid congeners produced by strain MCTG107b was characterized by HPLC-MS operating in the positive mode. The observed products possessed $\mathrm{m} / \mathrm{z}$ values that corresponded to values for known rhamnolipids, indicating that the biosurfactant synthesised by strain MCTG107b was a mixture of rhamnolipid congeners. We identified a variety of separate rhamnolipid congeners present in purified cell-free supernatant extracts from culture samples of the strain (Table 2). These congeners included both mono- and di-rhamnolipids; however, there was an overwhelming preference toward the synthesis of di-rhamnolipid (95.39\% of total rhamnolipid abundance). The congener with the highest relative abundance $(52.45 \%)$ possessed an $m / z$ value of 651.73 . This value correlated with $\quad \alpha$-L-rhamnopyranosyl- $\alpha$-L-rhamnopyranosyl$\beta$-hydroxydecanoyl- $\beta$-hydroxydecanoate (Rha-Rha$\mathrm{C}_{10}-\mathrm{C}_{10}$ ) with a molecular weight of $650.79 \mathrm{Da}$. The next most abundantly synthesised congeners were Rha-Rha- $\mathrm{C}_{10}-\mathrm{C}_{10} \mathrm{CH}_{3}$ (23.07\%), Rha-Rha- $\mathrm{C}_{10}$ (5.13\%), Rha-Rha- $\mathrm{C}_{10}-\mathrm{C}_{12}$ (5.01\%), Rha-Rha- $\mathrm{C}_{10}-\mathrm{C}_{12} \mathrm{CH}_{3}$ (3.26\%) and $\mathrm{Rha}-\mathrm{C}_{14: 2}(3.18 \%)$ (Table 2). As rhamnolipid production has not been previously observed in any member of the Marinobacter genus, these data were further investigated for confirmatory evidence of this. For this, tandem-MS was performed on the major molecular ion shown to be synthesised by strain MCTG107b. Tandem-MS analysis of the compound with an $m / z$ of 651.73 revealed the detection of 'daughter' ions with molecular weights indicative of the fragmentation of Rha-Rha- $\mathrm{C}_{10}-\mathrm{C}_{10}$ (Fig. 3). These data, together with the phenotypic results and initial HPLC-MS and Nuclear Magnetic Resonance (NMR) spectroscopy analysis

Table 2 Composition of rhamnolipid congeners synthesised by Marinobacter sp. MCTG107b

\begin{tabular}{|c|c|c|c|c|c|}
\hline RT min & $m / z$ value & Compound & $\mathrm{Mw}(\mathrm{Da})$ & Molecular form & Relative \% \\
\hline \multicolumn{6}{|c|}{ Mono-rhamnolipid congeners } \\
\hline 14.8 & 387.22 & Rha- $C_{14: 2}$ & 386.48 & $\mathrm{C}_{20} \mathrm{H}_{34} \mathrm{O}_{7}$ & 3.18 \\
\hline 21.5 & 533.46 & Rha $-C_{10}-C_{12} /$ Rha $-C_{12}-C_{10}$ & 532.71 & $\mathrm{C}_{28} \mathrm{H}_{52} \mathrm{O}_{9}$ & 0.22 \\
\hline 24.2 & 503.47 & Rha- $C_{10}-C_{10: 1}$ & 502.64 & $\mathrm{C}_{26} \mathrm{H}_{46} \mathrm{O}_{9}$ & 0.27 \\
\hline 26.9 & 561.52 & Rha $-C_{12}-C_{12} /$ Rha $-C_{10}-C_{14}$ & 560.76 & $\mathrm{C}_{30} \mathrm{H}_{56} \mathrm{O}_{9}$ & 0.94 \\
\hline Subtota & & & & & 4.61 \\
\hline \multicolumn{6}{|c|}{ Di-rhamnolipid congeners } \\
\hline 4.6 & 453.27 & Rha-Rha- $C_{8}$ & 452.49 & $\mathrm{C}_{20} \mathrm{H}_{36} \mathrm{O}_{11}$ & 1.95 \\
\hline 12.7 & 480.39 & Rha-Rha- $C_{10}$ & 480.55 & $\mathrm{C}_{22} \mathrm{H}_{40} \mathrm{O}_{11}$ & 5.13 \\
\hline 22.1 & 537.45 & Rha-Rha- $C_{14}$ & 536.65 & $\mathrm{C}_{26} \mathrm{H}_{48} \mathrm{O}_{11}$ & 0.21 \\
\hline 31.0 & 649.71 & Rha-Rha- $C_{10}-C_{10: 1} /$ Rha-Rha- $C_{10: 1}-C_{10}$ & 648.74 & $\mathrm{C}_{32} \mathrm{H}_{56} \mathrm{O}_{13}$ & 2.85 \\
\hline 32.1 & 651.73 & Rha-Rha- $C_{10}-C_{10}$ & 650.79 & $\mathrm{C}_{34} \mathrm{H}_{58} \mathrm{O}_{13}$ & 52.45 \\
\hline 32.8 & 677.77 & Rha-Rha- $C_{10}-C_{12: 1}$ & 676.83 & $\mathrm{C}_{33} \mathrm{H}_{60} \mathrm{O}_{13}$ & 1.06 \\
\hline 33.0 & 665.77 & Rha-Rha- $\mathrm{C}_{10}-\mathrm{C}_{10}-\mathrm{CH}_{3}$ & 664.82 & $\mathrm{C}_{42} \mathrm{H}_{60} \mathrm{O}_{13}$ & 23.07 \\
\hline 34.5 & 803.54 & Decenoyl-Rha-Rha- $C_{10}-C_{10: 1}$ & 801.01 & $\mathrm{C}_{35} \mathrm{H}_{72} \mathrm{O}_{11}$ & 0.40 \\
\hline 35.1 & 679.78 & Rha-Rha- $\mathrm{C}_{10}-\mathrm{C}_{12} /$ Rha-Rha- $\mathrm{C}_{12}-\mathrm{C}_{10}$ & 678.84 & $\mathrm{C}_{35} \mathrm{H}_{64} \mathrm{O}_{13}$ & 5.01 \\
\hline 37.2 & 693.90 & Rha-Rha- $\mathrm{C}_{10}-\mathrm{C}_{12}-\mathrm{CH}_{3} /$ Rha-Rha- $\mathrm{C}_{12}-\mathrm{C}_{10}-\mathrm{CH}_{3}$ & 692.80 & $\mathrm{C}_{35} \mathrm{H}_{64} \mathrm{O}_{13}$ & 3.26 \\
\hline Subtotal & & & & & 95.39 \\
\hline
\end{tabular}




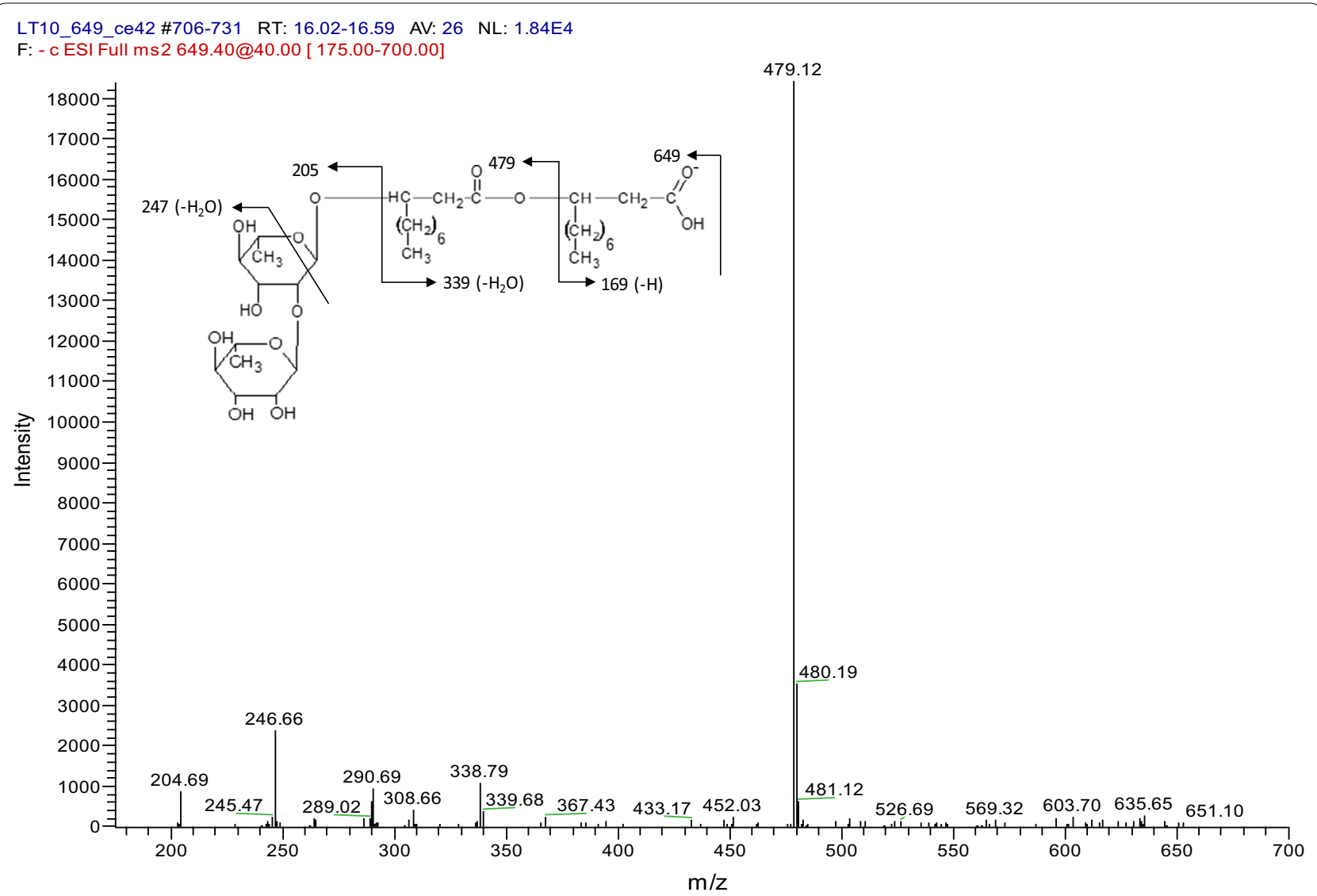

Fig. 3 HPLC-MS-MS profile of daughter products resulting from the fragmentation of a molecular ion with an $\mathrm{m} / \mathrm{z}$ of 651.73 , observed in a previous HPLC-MS analysis to be the predominant compound in supernatant extracts from Marinobacter sp. MCTG107b. The observed products corresponded to the predicted molecular weights of the fragmentation of di-rhamnolipid Rha-Rha- $C_{10}-C_{10}$. Fragments below $m / z 205$ were not detected due to sensitivity of the instrument

(data not shown), confirms the synthesis of rhamnolipid by Marinobacter sp. MCTG107b.

\section{Assessment of virulence using the Galleria mellonella infection model}

The potential virulence of Marinobacter sp. MCTG107b was assessed and compared to that of the rhamnolipid producing opportunistic pathogen $P$. aeruginosa using the G. mellonella infection model. P. aeruginosa PAO1 killed $100 \%$ of the infected larvae 24 h post inoculation with as little as $1000 \mathrm{CFU}$. When larvae were inoculated with an equal CFU count of Marinobacter sp. MCTG107b or with sterile PBS, the larvae showed 97\% survival $48 \mathrm{~h}$ post inoculation, with only one larva dying in each experimental group at 22 and $20 \mathrm{~h}$ post inoculation respectively (Fig. 4). Similar survival rates were also observed when larvae were inoculated with significantly

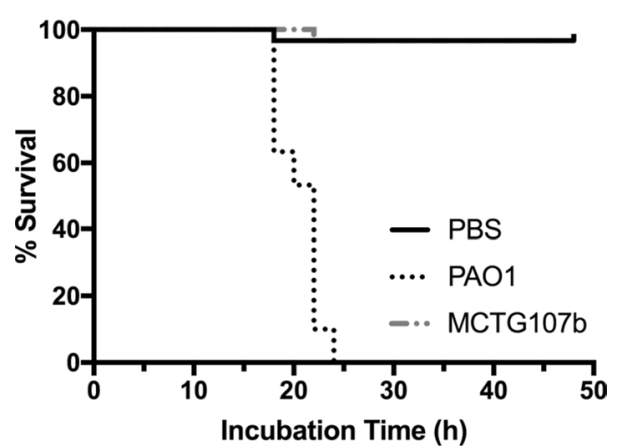

Fig. 4 Kaplan-Meier plot showing percentage survival of Galleria mellonella larvae after inoculation with either Marinobacter sp. MCTG107b or P. aeruginosa PAO1. Within a $48 \mathrm{~h}$ incubation there was no significant mortality observed after infection with cells of strain MCTG107b as opposed to infection with strain PAO1 where 100\% mortality was observed following $24 \mathrm{~h}$ incubation. Additionally, no significant mortality was observed in larvae inoculated with the carrier control buffer (PBS). $n=30$ (pooled from $3 \times$ duplicate experiments) 
higher doses of Marinobacter sp. MCTG107b (up to $10,000 \mathrm{CFU}$ in $20 \mu \mathrm{L}$ ) (data not shown).

\section{Discussion}

Marine bacteria are reported to secrete surface-active molecules that can interact with hydrocarbons to increase the emulsification of the hydrocarbon molecules in seawater to enable these, and also non-biosurfactant producing bacteria to access these molecules for uptake and use as a source of carbon and energy [39, 40]. Marinobacter, a genus of Gammaproteobacteria, has previously been shown capable of utilising hydrocarbons as growth substrates by producing biosurfactants or bioemulsifier $[41,42]$. In the present study, five marine bacterial strains, which were originally isolated for their ability to grow on and degrade PAHs, were identified to belong to the genus Marinobacter based on 16S rDNA gene sequencing. These isolates were screened to evaluate their potential as biosurfactant producers when cultivated in marine media using rapeseed oil as a carbon source. Many previous studies have reported that the inclusion of peptone in marine media is essential for biosurfactant synthesis [43, 44]. Here we show that in response to adding rapeseed oil to peptone containing ZM/1 media, these five Marinobacter strains displayed varying phenotypic responses which were indicative of biosurfactant synthesis. Phenotypic comparison of all these strains showed that Marinobacter sp. MCTG107b, isolated from sea surface water samples off the coast of Oregon, USA, showed maximum reduction in the surface tension of cell-free supernatant fractions. As the ability to reduce the surface tension of cell-free supernatant is a key phenotypic marker of lowmolecular weight biosurfactant synthesis, this strain was selected for further investigation [45].

Considering that subtle changes in salinity and $\mathrm{pH}$ can exert an important effect on microbial communities in the marine environment $[46,47]$, these parameters were tested for their influence on the growth of strain MCTG107b and its production of biosurfactant. Indeed, several studies have shown the effect of these two parameters on bacterial biosurfactant synthesis. For example, Bacillus subtilis N3-1P, isolated from brewery waste, reduced the surface tension of the culture medium to the greatest extent at a $\mathrm{pH}$ of 6.41 when compared to a range of other media pHs [48]. A thermophilic and halo-tolerant strain of $P$. aeruginosa, isolated from oilcontaminated soil, produced biosurfactant when cultured in media containing a salinity range of $0-6 \%(\mathrm{w} / \mathrm{v})$ [49]. In contrast Bacillus licheniformis BAS50, isolated from a deep oil well, produced biosurfactant when using a salinity of up to $13 \% \mathrm{NaCl}$, which is a salinity equivalent to that present in many petroleum reservoirs [50]. Based on these previous studies, we carried out FFD modelling to identify optimal concentrations of salinity and $\mathrm{pH}$ for the maximal production of biosurfactant by strain MCTG107b, and which revealed that a $\mathrm{pH}$ range of 5.0-6.8 and salinity concentration of $22.5-40 \mathrm{~g} \mathrm{~L}^{-1}$ were optimal. Interestingly, while salinity was the dominant factor affecting the reduction of surface tension, the strain preferred an acidic $\mathrm{pH}$ for increased biosurfactant production. This was at the expense of bacterial growth, indicating that when the culture medium was alkaline, cells were directed to the production of cellular biomass over biosurfactant synthesis. Whilst not within the focus of this study, future work could be directed to explore this $\mathrm{pH}$-mediated biosurfactant response and whether it is transposable to the global ocean in order to predict microbial biosurfactant production under future climate change conditions, such as ocean acidification. The profile of a marked fall in surface tension during the exponential growth phase coupled with a sustained low surface tension during stationary phase indicated the biosurfactant produced by strain MCTG107b is likely a secondary metabolite, as has been similarly reported for P. aeruginosa [51], B. thailandensis [23] and marine Pseudomonas sp. MCTG214(3b1) [20].

Orcinol assay yielded $150 \mu \mathrm{g} \mathrm{mL} \mathrm{L}^{-1}$ of rhamnolipid in the cell free supernatant. Orcinol assay is a colorimetric method for the rapid indication of rhamnolipid in the fermentation broth. However, the limitation of orcinol method is that, it is not specific for rhamnose and impurities present in the sample might interfere with the actual yield. We followed the criteria suggested by Irorere et al. [35] to quantify biosurfactant yield gravimetrically. The final yield of rhamnolipid was $740 \pm 28.3 \mathrm{mg} \mathrm{L}^{-1}$ from the SPE purified sample. SPE purification of crude biosurfactant removed excess lipids from the sample which gave an absolute quantification of rhamnolipid yield. Similarly, Perfumo et al. [51] reported purified rhamnolipid yields in the range of $0.8-1.7 \mathrm{~g} \mathrm{~L}^{-1}$ from $P$. aeruginosa strains. While, the orcinol assay provided an overestimation of rhamnolipids at 7.7-9.5 $\mathrm{g} \mathrm{L}^{-1}$.

The chemical characterisation of the purified biosurfactant synthesised by strain MCTG107b was achieved using HPLC-MS-a methodology demonstrated to be the most effective for identifying biosurfactant compounds [35]. We identified 14 separate rhamnolipid congeners with Rha-Rha- $\mathrm{C}_{10}-\mathrm{C}_{10}(\mathrm{~m} / z$ 651) being the most abundant $(52.45 \%)$. Furthermore, elucidation of this major molecular ion synthesised by Marinobacter sp. MCTG107b was performed by tandem-MS. When fragmented by MS-MS, the parent ion showed characteristic fragments at $m / z 479$ and $m / z 339$, which were in agreement with the previous tandem-MS analysis of RhaRha- $\mathrm{C}_{10}-\mathrm{C}_{10}$ performed by Zhao et al. [52]. The pattern of rhamnolipid congeners synthesised by this strain was 
highly similar to the rhamnolipid congeners that have been shown to be produced by both $P$. aeruginosa and marine Pseudomonas sp. MCTG214(3b1), but contrasts with $B$. thailandensis which produces an abundance of congeners with di-rhamnolipid containing $C_{14}[18,20$, 23]. The composition of rhamnolipid congeners greatly affects its properties. Mono-rhamnolipids have been reported to more effectively solubilise PAHs compared to di-rhamnolipids. However, di-rhamnolipids have better rate of biodegradation than mono-rhamnolipids due to the slow release of PAH from the mono-rhamnolipid micelles [53]. This was seen with methyl esters of dirhamnolipid which were reported to be effective in promoting alkane degradation [54]. Interestingly, non-ionic rhamnolipids or methyl esters of di-rhamnolipid $\mathrm{C}_{10}-\mathrm{C}_{10}$ and novel methyl ester of di-rhamnolipid $\mathrm{C}_{10}-\mathrm{C}_{12}$ were also identified in this study. In this study a mono-rhamnolipid with single 3-hydroxy fatty acid chain $\mathrm{Rha}-\mathrm{C}_{14 \cdot 2}$ (3.18\%) was also detected which was previously reported in rhamnolipid produced by $P$. aeruginosa mutant MIG-N146 [55]. Under our experimental conditions, MCTG107b was able to produce diverse rhamnolipid congeners with aliphatic chains varying from $\mathrm{C}_{8}$ to $\mathrm{C}_{14}$ and few congeners with unsaturated bonds.

The biosynthesis of rhamnolipids in $P$. aeruginosa occurs in three enzymatic steps. In the first step, rhamnosyltransferase chain A RhlA (encoded by the rhlA gene), synthesizes a fatty acid dimer molecule from $\beta$-hydroxy fatty acid precursors [56]. The second step, RhlB rhamnosyltransferase chain B (encoded by the rhlB gene), produces mono-rhamnolipids by covalently bonding the previously synthesised precursor molecule and dTDP-L-rhamnose [57]. The final step, RhlC rhamnosyltransferase II (encoded by rhlC gene), utilises mono-rhamnolipids synthesised by RhlA and RhlB as a substrate, adding a second dTDP-L-rhamnose moiety to produce di-rhamnolipids [58]. The rhamnolipid congener profile produced by Marinobacter sp. MCTG107b is predominantly skewed toward the synthesis of di-rhamnolipids, whereas mono-rhamnolipids were only found in much smaller concentrations. Although a prevalence toward di-rhamnolipid synthesis has been observed in $P$. aeruginosa, and to an even greater extent in Burkholderia species, the abundance of di-rhamnolipid versus mono-rhamnolipid in Marinobacter sp. MCTG107b was significantly higher than that previously observed with other rhamnolipid-producing organisms. In P. aeruginosa a single copy of rhlA and $r h l B$ are located in an operon alongside genes encoding an AHL-mediate quorum sensing system. The $r h l C$ gene is located approx. $1 \mathrm{Mbp}$ down stream of this operon [51]. A contrasting arrangement was observed in $B$. thailandensis which has two identical and functional operons containing orthologues of each rhamnolipid synthesis gene ( $r h l A, r h l B$ and $r h l C)$, possessing only $40 \%$ sequence similarity to those of $P$. aeruginosa [23, 59]. Therefore, Burkholderia species can simultaneously express $r h l B$ and $r h l C$ favouring the immediate addition of the second rhamnosyl group to the produced mono-rhamnolipid [59].

We therefore postulate that a biosynthetic pathway similar to that observed in Burkholderia might be present here and accounting for the higher ratio of di-rhamnolipid to mono-rhamnolipid congeners. The biosynthetic pathway of rhamnolipid synthesis by Marinobacter sp. has however presented a paradox. Although not shown here, we have carried out additional PCR screening for $r h l A$, rhlB and $r h l C$ using degenerate primers designed from multiple sequence alignments of both $P$. aeruginos $a$ and $B$. thailandensis sequences. To date, this approach has failed to amplify any DNA sequence which could be involved in rhamnolipid synthesis gene orthologues. Our finding that other similar Marinobacter strains fail to synthesise rhamnolipids suggests that the acquisition of rhamnolipid biosynthesis genes by Marinobacter sp. MCTG107b may have occurred through lateral gene transfer from an unrelated rhamnolipid producing species, as has been observed previously in other rhamnolipid synthesising bacteria [60]. However, based on the un-relatedness of Marinobacter sp. MCTG107b to previously reported rhamnolipid producers and the significant sequence differences between the $P$. aeruginos $a$ and $B$. thailandensis synthases, we conclude that rhamnolipid synthesis in this strain is being catalysed by enzymes with significantly different peptide sequences to either of these other species. This sequence difference is also present at the genetic level, accounting for the reasons why our screening protocol failed [23, 59]. Additionally, the observation of mono-rhamnolipid congeners only possessing a single fatty acid side chain being synthesised by this strain corroborates this conclusion since RhlA in both $P$. aeruginosa and Burkholderia species utilises fatty acid dimers as a substrate for rhamnolipid synthesis $[56,59]$. To further investigate the mechanisms of rhamnolipid biosynthesis, we recently obtained the complete genome sequence of Marinobacter sp. MCTG107b. We are therefore, currently in the process of carrying out comparative genomic analysis with various other Marinobacter strains, with the aim of identifying putative genetic candidates for rhamnolipid biosynthesis.

\section{Conclusions}

Marinobacter sp. MCTG107b, isolated from the marine environment, has the ability to synthesise a wide variety of rhamnolipid congeners. To the best of our knowledge, rhamnolipid production has not been previously 
observed in any member of the genus Marinobacter. Therefore, the results presented here expand the list of known rhamnolipid producing bacterial taxa to include Marinobacter; a genus of marine bacteria that shows little to no association with human pathogenicity. Although the major hurdle of low production yield remains, synthesis of rhamnolipids from novel, non-pathogenic marine species, such as Marinobacter sp. MCTG107b, is promising for the scale-up in bioprocessing industry or to provide genetic resources for metabolic engineering for the production of specific rhamnolipid congeners.

\section{Supplementary information}

Supplementary information accompanies this paper at https://doi. org/10.1186/s12934-019-1216-8.

Additional file 1: Fig. S1. Effect of various sea salt concentrations, $\mathrm{pH}$, temperature and nitrogen source on the growth of Marinobacter sp. MCTG107b over a period of $96 \mathrm{~h}$. Growth curves show the $\mathrm{OD}_{600}$ of strain MCTG107b during growth at different (A) salinities (5.0 to $40 \mathrm{~g} \mathrm{~L}^{-1}$ ), (B) pH values (4.0 to 8.5), or (C) temperatures $\left(25^{\circ} \mathrm{C}, 28^{\circ} \mathrm{C}, 30^{\circ} \mathrm{C}, 37^{\circ} \mathrm{C}\right)$. Fig. $\mathbf{S 2}$. HPLC-MS chromatogram for rhamnolipids produced by Marinobacter sp. MCTG107b. The MS was operated in the negative mode. Main intensities in the chromatogram were Rha-Rha- $\mathrm{C}_{10}-\mathrm{C}_{10}$ and Rha-Rha- $\mathrm{C}_{10}-\mathrm{C}_{10}-\mathrm{CH}_{3}$. Table S1. Full $2^{2}$ factorial design with $\mathrm{pH}$ and salt as independent variables using surface tension and biosurfactant yield as response variables. Surface tension and biosurfactant yield according to full factorial design after 96 h of shake-flask study of Marinobacter sp. MCTG107b. Table S2. Analysis of variance (ANOVA) for response variables surface tension and biosurfactant yield by Marinobacter sp. MCTG107b.

\section{Abbreviations}

PAHs: polycyclic aromatic hydrocarbons; MEOR: microbial enhanced oil recovery; $\beta$-OH-FA: $\beta$-hydroxy fatty acids; DoE: design of experiments; FFD: full factorial design; gDNA: genomic DNA; El: emulsification index; SPE: solid phase extraction; HPLC-MS: high performance liquid chromatography-mass spectrometry; NMR: Nuclear Magnetic Resonance.

\section{Acknowledgements}

The authors would like to acknowledge the support of the European Union Framework Programme for Research and Innovation, Horizon 2020 under Grant agreement No. 635340 MARISURF. VUI would also like to acknowledge the support of an Ulster University Vice Chancellors Research Scholarship. The authors would like to thank Diego Cobice and Sarah Dobbin for their technical support during the spectrometric analysis.

\section{Authors' contributions}

LT designed the experiments, executed experimental work, analyzed data and drafted the manuscript. MST performed phylogenetic identification, virulence assessment of the strain and assisted with the drafting of the manuscript. AZ performed the HPLC-MS analysis. KS assisted in phenotypic screening of strains. TG, VUI, and GAS assisted in reviewing the manuscript. RM and IMB designed and supervised the studies. All authors read and approved the final manuscript.

\section{Availability of data and materials}

All data generated or analysed during this study are included in this published article and its additional files.

Ethics approval and consent to participate

Not applicable.
Consent for publication

Not applicable.

\section{Competing interests}

The authors declare that they have no competing interests.

\section{Author details}

${ }^{1}$ School of Biomedical Sciences, Ulster University, Coleraine BT521SA, Northern Ireland, UK. ${ }^{2}$ Department of Pharmacy, University of Patras, 26504 Patras, Greece. ${ }^{3}$ Institute of Mechanical, Process \& Energy Engineering, School of Engineering \& Physical Sciences, Heriot-Watt University, Edinburgh EH14 4AS, UK.

Received: 10 August 2019 Accepted: 24 September 2019

Published online: 10 October 2019

\section{References}

1. Naughton PJ, Marchant R, Naughton V, Banat IM. Microbial biosurfactants: current trends and applications in agricultural and biomedical industries. J Appl Microbiol. 2019;127:12-28.

2. Bijlsma R, Loeschcke V. Environmental stress, adaptation and evolution: an overview. J Evol Biol. 2005;18:744-9.

3. de Carvalho CCCR. Marine biofilms: a successful microbial strategy with economic implications. Front Mar Sci. 2018;5:126.

4. Decho AW, Gutierrez T. Microbial extracellular polymeric substances (EPSs) in ocean systems. Front Microbiol. 2017;8:922.

5. Gutierrez T, Singleton DR, Berry D, Yang T, Aitken MD, Teske A. Hydrocarbon-degrading bacteria enriched by the deepwater horizon oil spill identified by cultivation and DNA-SIP. ISME J. 2013;7:2091-104.

6. Suja LD, Summers S, Gutierrez T. Role of EPS, dispersant and nutrients on the microbial response and MOS formation in the Subarctic Northeast Atlantic. Front Microbiol. 2017;8:676.

7. Antoniou E, Fodelianakis S, Korkakaki E, Kalogerakis N. Biosurfactant production from marine hydrocarbon-degrading consortia and pure bacterial strains using crude oil as carbon source. Front Microbiol. 2015;6:274.

8. Gutierrez T, Berry D, Yang T, Mishamandani S, McKay L, Teske A, Aitken MD. Role of bacterial exopolysaccharides (EPS) in the fate of the oil released during the Deepwater Horizon Oil Spill. PLoS ONE. 2013;8:e67717.

9. Marchant R, Banat IM. Biosurfactants: a sustainable replacement for chemical surfactants? Biotechnol Lett. 2012;34:1597-605.

10. Marchant R, Banat IM. Microbial biosurfactants: challenges and opportunities for future exploitation. Trends Biotechnol. 2012;30:558-65.

11. Perfumo A, Banat IM, Marchant R. Going green and cold: biosurfactants from low-temperature environments to biotechnology applications. Trends Biotechnol. 2018;36:277-89.

12. Pamp SJ, Tolker-Nielsen T. Multiple roles of biosurfactants in structural biofilm development by Pseudomonas aeruginosa. J Bacteriol. 2007;189:2531-9.

13. Dey G, Bharti R, Dhanarajan G, Das S, Dey KK, Kumar BN, Sen R, Mandal M. Marine lipopeptide Iturin A inhibits Akt mediated GSK3beta and FoxO3a signaling and triggers apoptosis in breast cancer. Sci Rep. 2015;2015(5):10316.

14. Kiran GS, Sabarathnam B, Selvin J. Biofilm disruption potential of a glycolipid biosurfactant from marine Brevibacterium casei. FEMS Immunol Med Microbiol. 2010;2010(59):432-8.

15. Banat IM, De Rienzo MA, Quinn GA. Microbial biofilms: biosurfactants as antibiofilm agents. Appl Microbiol Biotechnol. 2014;98:9915-29.

16. Mireles JR 2nd, Toguchi A, Harshey RM. Salmonella enterica serovar Typhimurium swarming mutants with altered biofilm-forming abilities: surfactin inhibits biofilm formation. J Bacteriol. 2001;183:5848-54.

17. Fracchia L, Banat J, Cavallo M, Ceresa C, Banat I. Potential therapeutic applications of microbial surface-active compounds. AIMS Bioeng. 2015;2:144-62.

18. Abdel-Mawgoud AM, Lepine F, Deziel E. Rhamnolipids: diversity of structures, microbial origins and roles. Appl Microbiol Biotechnol. 2010;86:1323-36.

19. Banat IM, Satpute SK, Cameotra SS, Patil R, Nyayanit NV. Cost effective technologies and renewable substrates for biosurfactants' production. Front Microbiol. 2014;5:697. 
20. Twigg MS, Tripathi L, Zompra A, Salek K, Irorere VU, Gutierrez T, Spyroulias GA, Marchant R, Banat IM. Identification and characterisation of short chain rhamnolipid production in a previously uninvestigated, non-pathogenic marine pseudomonad. Appl Microbiol Biotechnol. 2018;102:8537-49.

21. Kristoffersen V, Rama T, Isaksson J, Andersen JH, GerwickWH, Hansen E. Characterization of rhamnolipids produced by an arctic marine bacterium from the Pseudomonas fluorescence group. Mar Drugs. 2018;2018(16):163.

22. Haussler S, Nimtz M, Domke T, Wray V, Steinmetz I. Purification and characterization of a cytotoxic exolipid of Burkholderia pseudomallei. Infect Immun. 1998;66:1588-93.

23. Funston SJ, Tsaousi K, Rudden M, Smyth TJ, Stevenson PS, Marchant R, Banat IM. Characterising rhamnolipid production in Burkholderia thailandensis E264, a non-pathogenic producer. Appl Microbiol Biotechnol. 2016;100:7945-56.

24. Whitman WB, Coleman DC, Wiebe WJ. Prokaryotes: the unseen majority. Proc Natl Acad Sci USA. 1998;95:6578-83.

25. Tripathi L, Irorere VU, Marchant R, Banat IM. Marine derived biosurfactants: a vast potential future resource. Biotechnol Lett. 2018;40:1441-57.

26. Brumano LP, Antunes FAF, Souto SG, Dos Santos JC, Venus J, Schneider R, da Silva SS. Biosurfactant production by Aureobasidium pullulans in stirred tank bioreactor: new approach to understand the influence of important variables in the process. Bioresour Technol. 2017;243:264-72.

27. Deepika KV, Kalam S, Ramu Sridhar P, Podile AR, Bramhachari PV. Optimization of rhamnolipid biosurfactant production by mangrove sediment bacterium Pseudomonas aeruginosa KVD-HR42 using response surface methodology. Biocatal Agric Biotechnol. 2016;5:38-47.

28. Pereira AG, Pacheco GJ, Tavares LF, Neves BC, Kronemberger FdA, Reis RS, Freire DMG. Optimization of biosurfactant production using waste from biodiesel industry in a new membrane assisted bioreactor. Process Biochem. 2013;48:1271-8.

29. Zobell CE. Studies on marine bacteria. I. The cultural requirements of heterotrophic aerobes. J Mar Res. 1941;4:41-75.

30. Morales SE, Cosart TF, Johnson JV, Holben WE. Extensive phylogenetic analysis of a soil bacterial community illustrates extreme taxon evenness and the effects of amplicon length, degree of coverage, and DNA fractionation on classification and ecological parameters. Appl Environ Microbiol. 2009;75:668-75.

31. Muhling M, Woolven-Allen J, Murrell JC, Joint I. Improved group-specific PCR primers for denaturing gradient gel electrophoresis analysis of the genetic diversity of complex microbial communities. ISME J. 2008;2:379-92.

32. du Nouy PL. An interfacial tensiometer for universal use. J Gen Physiol. 1925;7:625-31.

33. Cooper DG, Goldenberg BG. Surface-active agents from two Bacillus species. Appl Environ Microbiol. 1987;53:224-9.

34. Smyth TJP, Rudden M, Tsaousi K, Marchant R, Banat IM. Protocols for the detection and chemical characterisation of microbial glycolipids. In: McGenity TJ, Timmis KN, Nogales B, editors. Hydrocarbon and lipid microbiology protocols: biochemical methods. Berlin: Springer; 2016. p. 29-60. https://doi. org/10.1007/8623_2014_25.

35. Irorere VU, Tripathi L, Marchant R, McClean S, Banat IM. Microbial rhamnolipid production: a critical re-evaluation of published data and suggested future publication criteria. Appl Microbiol Biotechnol. 2017;101:3941-51.

36. Marchant R, Banat IM. Protocols for measuring biosurfactant production in microbial cultures. In: McGenity TJ, Timmis KN, Nogales B, editors. Hydrocarbon and lipid microbiology protocols: activities and phenotypes. Berlin: Springer; 2017. p. 119-28. https://doi.org/10.1007/8623_2014_10.

37. Miles AA, Misra SS, Irwin JO. The estimation of the bactericidal power of the blood. Epidemiol Infect. 2009;38:732-49.

38. Hill L, Veli N, Coote PJ. Evaluation of Galleria mellonella larvae for measuring the efficacy and pharmacokinetics of antibiotic therapies against Pseudomonas aeruginosa infection. Int J Antimicrob Agents. 2014;43:254-61.

39. De Almeida DG, Soares Da Silva RC, Luna JM, Rufino RD, Santos VA, Banat IM, Sarubbo LA. Biosurfactants: promising molecules for petroleum biotechnology advances. Front Microbiol. 2016;7:1718.

40. Franzetti A, Tamburini E, Banat IM. Applications of biological surface active compounds in remediation technologies. Adv Exp Med Biol. 2010;672:121-34

41. Bonin P, Vieira C, Grimaud R, Militon C, Cuny P, Lima O, Guasco S, Brussaard CP, Michotey V. Substrates specialization in lipid compounds and hydrocarbons of Marinobacter genus. Environ Sci Pollut Res Int. 2015;22:15347-59.
42. Raddadi N, Giacomucci L, Totaro G, Fava F. Marinobacter sp. from marine sediments produce highly stable surface-active agents for combatting marine oil spills. Microb Cell Fact. 2017;16:186.

43. GutierrezT, Biller DV, Shimmield T, Green DH. Metal binding properties of the EPS produced by Halomonas sp. TG39 and its potential in enhancing trace element bioavailability to eukaryotic phytoplankton. Biometals. 2012;25:1185-94.

44. Rocha MV, Souza MC, Benedicto SC, Bezerra MS, Macedo GR, Pinto GA, Goncalves LR. Production of biosurfactant by Pseudomonas aeruginosa grown on cashew apple juice. Appl Biochem Biotechnol. 2007;137-140:185-94.

45. Banat IM, Franzetti A, Gandolfi I, Bestetti G, Martinotti MG, Fracchia L, Smyth TJ, Marchant R. Microbial biosurfactants production, applications and future potential. Appl Microbiol Biotechnol. 2010;87:427-44.

46. Oren A. Microbial life at high salt concentrations: phylogenetic and metabolic diversity. Saline Syst. 2008;4:1-13.

47. Krause E, Wichels A, Gimenez L, Lunau M, Schilhabel MB, Gerdts G. Small changes in $\mathrm{pH}$ have direct effects on marine bacterial community composition: a microcosm approach. PLoS ONE. 2012;7:e47035.

48. Moshtagh B, Hawboldt K, Zhang B. Optimization of biosurfactant production by Bacillus subtilis N3-1P using the brewery waste as the carbon source. Environ Technol. 2018. https://doi.org/10.1080/09593330.2018.14735 02:1-10.

49. Kumar M, León V, De Sisto Materano A, Ilzins O, Luis L. Biosurfactant production and hydrocarbon-degradation by halotolerant and thermotolerant Pseudomonas sp. World J Microbiol Biotechnol. 2008;24:1047-57.

50. Yakimov MM, Timmis KN, Wray V, Fredrickson HL. Characterization of a new lipopeptide surfactant produced by thermotolerant and halotolerant subsurface Bacillus licheniformis BAS50. Appl Environ Microbiol. 1995;61:1706-13.

51. Perfumo A, Rudden M, Smyth TJ, Marchant R, Stevenson PS, Parry NJ, Banat IM. Rhamnolipids are conserved biosurfactants molecules: implications for their biotechnological potential. Appl Microbiol Biotechnol. 2013;97:7297-306.

52. Zhao J, Wu Y, Alfred AT, Xin X, Yang S. Chemical structures and biological activities of rhamnolipid biosurfactants produced by Pseudomonas aeruginosa M14808. J Chem Phar Res. 2013;5:177-82.

53. Zhang Y, Maier WJ, Miller RM. Effect of rhamnolipids on the dissolution, bioavailability, and biodegradation of phenanthrene. Environ Sci Technol. 1997;31:2211-7.

54. Zhang Y, Miller RM. Effect of rhamnolipid (biosurfactant) structure on solubilization and biodegradation of $n$-alkanes. Appl Environ Microbiol. 1995;1995(61):2247-51.

55. Guo YP, Hu YY, Gu RR, Lin H. Characterization and micellization of rhamnolipidic fractions and crude extracts produced by Pseudomonas aeruginosa mutant MIG-N146. J Colloid Interface Sci. 2009;331:356-63.

56. Zhu K, Rock CO. RhIA converts beta-hydroxyacyl-acyl carrier protein intermediates in fatty acid synthesis to the beta-hydroxydecanoyl-betahydroxydecanoate component of rhamnolipids in Pseudomonas aeruginosa. J Bacteriol. 2008;190:3147-54.

57. Deziel E, Lepine F, Milot S, Villemur R. rhIA is required for the production of a novel biosurfactant promoting swarming motility in Pseudomonas aeruginosa: 3-(3-hydroxyalkanoyloxy)alkanoic acids (HAAs), the precursors of rhamnolipids. Microbiology. 2003;149:2005-13.

58. Rahim R, Ochsner UA, Olvera C, Graninger M, Messner P, Lam JS, SoberonChavez G. Cloning and functional characterization of the Pseudomonas aeruginosa rh/C gene that encodes rhamnosyltransferase 2, an enzyme responsible for di-rhamnolipid biosynthesis. Mol Microbiol. 2001;40:708-18.

59. Dubeau D, Deziel E, Woods DE, Lepine F. Burkholderia thailandensis harbors two identical rh/ gene clusters responsible for the biosynthesis of rhamnolipids. BMC Microbiol. 2009;9:263.

60. Toribio J, Escalante AE, Soberón-Chávez G. Rhamnolipids: production in bacteria other than Pseudomonas aeruginosa. Eur J Lipid Sci Tech. 2010;112:1082-7.

\section{Publisher's Note}

Springer Nature remains neutral with regard to jurisdictional claims in published maps and institutional affiliations. 